\title{
POSTER: On the Overhead of Using Zero-Knowledge Proofs for Electric Vehicle Authentication
}

\author{
David Gabay, Mumin Cebe and Kemal Akkaya \\ Dept. of Electrical and Computer Engineering, Florida International University \\ Miami, FL, USA 33174 \\ \{dagaba002,mcebe,kakkaya\}@fiu.edu
}

\begin{abstract}
As Electric Vehicles (EVs) are becoming widely available, their secure management is crucial to fully enable their potential. For instance, for convenient charging, they may require quick authentication with the charging stations while they are on the go. As charging is frequently needed, exposing one's charging frequency to the stations may risk the exposure of privacy for the EV driver. Therefore, a mechanism is needed to hide EV information. In this paper, we propose using zero-knowledge proofs to achieve this goal. While zero-knowledge proofs can provide anonymous authentication, they require computation for generation of witnesses. Therefore, we assess the overhead of generating a witness and proof computation at the resource constrained on-board units (OBUs) which are deployed on EVs that utilize wireless communications for scheduling. The results indicate that computation overhead is minimal and can be delployed on resource contrained devices.
\end{abstract}

\section{CCS CONCEPTS}

- Security and privacy $\rightarrow$ Privacy-preserving protocols; Mobile and wireless security;

\section{KEYWORDS}

EVs, charging, privacy, zkSNARKs, zero-knowledge proof.

\section{ACM Reference format:}

David Gabay, Mumin Cebe and Kemal Akkaya. 2019. POSTER: On the Overhead of Using Zero-Knowledge Proofs for Electric Vehicle Authentication. In Proceedings of WiSec '19: ACM Conference on Security and Privacy in Wireless and Mobile Networks, Miami, FL, USA, May 15-17, 2019 (WiSec '19), 2 pages.

https://doi.org/10.1145/3317549.3326325

\section{INTRODUCTION}

EVs are a type of smart car that can use Vehicular to Infrastructure (V2I) technology to communicate with existing EV charging stations [1]. This is needed because the EV charging is time consuming process which can take hours depending on the speed of the charging station. Since EV charging stations may be occupied and charging is frequently needed due to low mileage of EVs, through V2I, the EVs can factor in traffic, battery status, and location to

Permission to make digital or hard copies of part or all of this work for personal or classroom use is granted without fee provided that copies are not made or distributed for profit or commercial advantage and that copies bear this notice and the full citation on the first page. Copyrights for third-party components of this work must be honored.

For all other uses, contact the owner/author(s)

WiSec '19, May 15-17, 2019, Miami, FL, USA

(C) 2019 Copyright held by the owner/author(s)

ACM ISBN 978-1-4503-6726-4/19/05 . \$ \$15.00

https://doi.org/10.1145/3317549.3326325 communicate with an upcoming EV charging station to schedule a charging slot.

However, in order for the EV to schedule the charging, it must authenticate with an Electric Vehicle Service Provider (EVSP). During the authentication and scheduling process, EVSPs will collect the battery status, location and time availability of EVs to provide the most suitable time slot. In this way, charging EVSP can learn the charging patterns, personal data, and location of the EV users. This raises privacy concerns as the EVSP can proceed to share the data with unwanted entities like insurance, marketers, etc [4]. To protect the EVs' privacy, the authentication and scheduling needs to be done in an anonymous manner. This means the EVSP should be able to authenticate the EVs while not recognizing which EV it is communicating. An alternative authentication method that can provide this service is through zero-knowledge proofs.

Zero-knowledge proofs [3] allow a user to prove the correctness of a statement using a proof without revealing the details of the statement or proof to the verifier. zkSNARKs [5] is an efficient variant of zero-knowledge proofs which allows the prover to prove his knowledge in a single message. By using zkSNARKs as the authentication mechanism, the EV would be able to prove that it is an authorized customer of the EVSP and receive charging scheduling without revealing its identity to the EVSP (See Fig. 1).

However, generating proofs in this way can put burden on resource constrained IoT devices such as OBUs on EVs. In this paper, we evaluate this overhead of generating a zero-knowledge proof particularly zkSNARKs using an underlying protocol Zokrates [2] for IoT devices. To simulate such a constrained device, we used a Raspberry Pi3 device and performed the computations required to generate a proof on this device. The results indicate that the proof generation can be still done in the order or milliseconds even though the proof generation function input size is large.

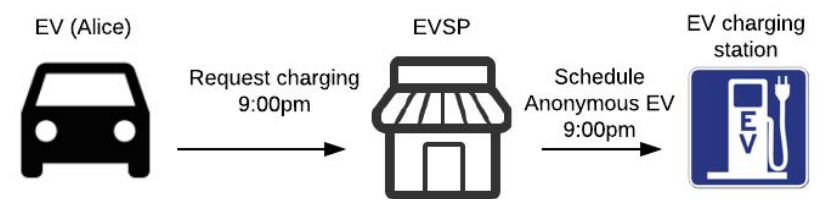

Figure 1: EV schedules charging time slot at a charging station as an authorized anonymous EV.

\section{PRELIMINARIES}

\subsection{Zero-Knowledge Proof and zkSNARKs}

In this section, we describe the different roles involved in a zeroknowledge proof. These roles are standard for all applications of zkSNARKs and exist in some form. 
- Trusted Third Party: The trusted third party is usually the EVSP and is in charge of registration of the user or prover The trusted third party will create a secret function which the prover will solve in order to authenticate himself to the EVSP. The trusted third party will also generate a contract with a verification key embedded to it. The contract is used by the verifier to verify the generated proofs.

- Prover: Prover is the client registered with the trusted third party. The prover will be in charge of solving the secret function in order to create a witness. Using the witness, it will generate a zero-knowledge proof attesting to its knowledge of the secret function, thus, authenticating itself.

- Verifier: The verifier is a third party which could verify the prover's proof without learning the details of the proof or the prover. The verifier uses the contract from the trusted third party to check the correctness of a proof.

In the zero knowledge commitment scheme, there exists a setup and verification phase. Let $U$ denote the user and prover, $t$ a trusted third party, $V$ the verifier, and $\pi$ an honestly generated proof. In order for a zero-knowledge proof to exist it must contain three properties [5]: 1) Completeness: $V$ always accepts an honestly generated proof $\pi$ from $U$; 2) Knowledge: A prover $U$ who does not know a secret will unlikely be able to generate a valid $\pi$ therefore unable to convince $V$; and 3) Zero Knowledge: A valid $\pi$ doesn't reveal any information about the secret. Protocol 1 provides the details for a zero-knowledge proof of knowledge.

Protocol 1 Zero knowledge commitment scheme

\section{(1) Setup}

(a) $t$ generates some secret function using some knowledge $U$ possess

(b) $U$ inputs values satisfying the condition of the function, returning the witness to $U$

(c) $U$ uses the witness to generate $\pi_{U}$

(2) Verification

(a) $U$ sends $\pi_{U}$ to $V$, who verifies the validity of $\pi_{U}$

(b) $V$ grants or denies access to $U$, without learning the contents of $\pi_{U}$

\subsection{Proposed Authentication Protocols}

Two protocols required to generate proofs are the witness attestation and proof generation phases. The witness attestation and proof generation phases should be done every time a user wishes to authenticate himself.

2.2.1 Witness attestation. Witness generation is done by the prover and is the process of finding variables that when used as input will satisfy the secret function. The secret function accepts private inputs as well as optional public inputs, that are added for complexity. This witness along with a proving key obtained from the EVSP will then be used in the proof generation.

2.2.2 Proof Generation. During this phase, the prover uses the distributed proving key and the witness to generate a proof. The proving key is a public key obtained from the EVSP during registration. The proof is later submitted to the verifier as authentication. The proving key, or witness cannot be inferred during verification.

\section{EVALUATIONS}

To evaluate the performance of utilizing a zkSNARK as authentication for an EV, we analyzed the witness and proof generation phases. Zokrates is the underlying protocol which contains to commands, compute-witness, and generate-proof. To simulate a real-life EV we use a Raspberry PI 3 model B V1.2 (Quad Cortex A53 @ 1.2GHz, 1GB SDRAM, $4000 \mathrm{~Hz}$ Videocore IV). We evaluate the performance of the protocol based on time and the size of the proof.

The Zokrates protocol converts a high level language program into Rank 1 Constraint Systems (R1CS) during a compilation phase. Each R1CS is composed of a number of constraints by which we measure complexity of a program being proven. In our experiments, we used different secret witness functions with different \# of inputs and constraints as shown in Table 1.

Table 1: Witness and proof generation time in seconds with functions that have 4,8 and 16 inputs, number of constraints, and the size of proof in bits.

\begin{tabular}{|c|c|c|c|c|}
\hline \# of Inputs & $\begin{array}{c}\text { \# of } \\
\text { Constraints }\end{array}$ & $\begin{array}{c}\text { Compute-witness } \\
\text { (seconds) }\end{array}$ & $\begin{array}{c}\text { Proof generation } \\
\text { (seconds) }\end{array}$ & $\begin{array}{c}\text { Proof size } \\
\text { (bits) }\end{array}$ \\
\hline $\mathbf{4}$ & 4 & 0.006 & 0.069 & 2294 \\
\hline $\mathbf{8}$ & 4 & 0.008 & 0.072 & 2294 \\
\hline $\mathbf{1 6}$ & 4 & 0.009 & 0.078 & 2294 \\
\hline $\mathbf{1 6}$ & 16 & 0.009 & 0.080 & 2294 \\
\hline $\mathbf{1 6}$ & 695 & 0.060 & 0.157 & 2294 \\
\hline
\end{tabular}

From these experiments, we concluded that the proposed authentication protocol using zkSNARKs does not carry a heavy burden on to the EV. The proof is 2294 bits in size making it feasible for transmitting to the EVSP. The EV can generate a witnesses and proofs between $0.075-0.217$ seconds depending on the complexity of the proofs, which can still provide almost real-time scheduling whenever there is a need.

\section{CONCLUSIONS}

When an EV authenticates to an EVSP in a request to schedule charging, its privacy is at risk from the EVSP. In this paper, we advocated an approach based on zero-knowledge proofs and assessed its overhead on resource contrained IoT devices such as OBUs on EVs. The results confirmed that ZkSNARKs can be a viable option that is not much differnt than existing traiditional signature based solutions.

\section{REFERENCES}

[1] Makena Coffman, Paul Bernstein, and Sherilyn Wee. 2017. Electric vehicles revisited: a review of factors that affect adoption. Transport Reviews 37, 1 (2017), 79-93. https://doi.org/10.1080/01441647.2016.1217282 arXiv:https://doi.org/10.1080/01441647.2016.1217282

[2] Jacob Eberhardt and Stefan Tai. 2018. ZoKrates-Scalable Privacy-Preserving Off-Chain Computations. In IEEE International Conference on Blockchain. IEEE.

[3] Shafi Goldwasser, Silvio Micali, and Charles Rackoff. 1989. The knowledge complexity of interactive proof systems. SIAM Journal on computing 18, 1 (1989), 186-208.

[4] Wenlin Han and Yang Xiao. 2016. Privacy preservation for V2G networks in smart grid: A survey. Computer Communications 91 (2016), 17-28.

[5] Hartwig Mayer. 2016. zk-SNARK explained: Basic Principles. (2016). 